Sādhanā Vol. 36, Part 3, June 2011, pp. 349-355. (c) Indian Academy of Sciences

\title{
Generation of intense soft X-rays from capillary discharge plasmas
}

\author{
Y B S R PRASAD*, S NIGAM, K ANEESH, S BARNWAL, \\ $P$ K TRIPATHI, P A NAIK, C P NAVATHE and P D GUPTA
}

Raja Ramanna Centre for Advanced Technology, Indore 452 013, India

e-mail: yprasad@rrcat.gov.in

MS received 18 March 2010; revised 14 June 2010; accepted 23 February 2011

\begin{abstract}
X-ray lasing through high voltage, high current discharges in gas filled capillaries has been demonstrated in several laboratories. This method gives highest number of X-ray photons per pulse. The fast varying current and the $\mathrm{j} \times \mathrm{B}$ magnetic force compress the plasma towards the axis forming a hot, dense, line plasma, wherein under appropriate discharge conditions lasing occurs. At Laser Plasma Division, RRCAT, a program on high voltage capillary discharge had been started. The system consists of a $400 \mathrm{kV}$ Marx bank, water line capacitor, spark gap and capillary chamber. The initial results of the emission of intense short soft X-ray pulses (5-10 ns) from the capillary discharge are reported.
\end{abstract}

Keywords. Capillary discharge; Marx bank; soft X-ray lasing.

\section{Introduction}

Generation of coherent X-ray radiation has attracted lot of attention worldwide due to its applications in diverse fields such as characterization of materials, studies in the areas of atomic physics, photo-chemistry, photo-physics, time resolved imaging, X-ray diffraction studies of biological and inorganic materials, diagnostics of high temperature plasmas, etc. (Filevich et al 2004; Filevich et al 2000). In addition, the excellent monochromaticity, directivity, coherence, the high brightness of the X-ray radiation helps in fabrication of integrated circuits of sub-micron scale lengths (articles from en.wikipedia.org and www.physorg.com).

Various approaches exist for generating coherent X-ray radiation which include X-ray lasing in laser produced plasmas (Hara et al 1989; Smith et al 1999), generation of higher harmonics using ultrashort laser pulses (Gupta \& Naik 2008; Salieres \& Lewenstein 2001), free electron lasers (Pellegrini \& Stohr 2003), etc. All these approaches are complimentary to each other in advantages and limitations. The X-ray lasers produced from laser produced plasmas have disadvantage of small gain volume resulting in lower flux, and no tunability. The high order harmonics which are partially tunable and have the problem of low conversion efficiency. The free electron lasers have the advantage of tunability but require generation of relativistic electron

*For correspondence 
beams. On the other hand, X-ray lasers produced using electrical discharge method in the gas filled capillaries give highest number of X-ray photons, as very large amount of electrical energy goes into the discharge.

X-ray lasers in the extreme ultra-violet (XUV or EUV) region using high voltage, high current capillary discharge Z-pinch plasmas have been demonstrated in several laboratories world-wide as a viable alternative to the above schemes (Ben-Kish et al 2001, Rocca et al 2003, Suckewer \& Jaegl'e 2009 and Tan \& Kwek 2007). A compact table-top capillary discharge laser (pulse energy $\sim 13 \mu \mathrm{J}$ with divergence $\sim 5.2 \mathrm{mrad}$ ) with $12 \mathrm{~Hz}$ repetition rate had been reported by Heinbuch et al (2005). X-ray lasers involving collisional excitation and recombination mechanisms, have been reviewed by Tallents (2003). Laser Plasma division, Raja Ramanna Centre for Advanced Technology (RRCAT) has started a project for generation of coherent radiation in the soft X-ray region $(46.9 \mathrm{~nm})$ from electrical discharges in argon gas. The construction of a capillary discharge system consisting a Marx bank, pulse shaping water capacitor, an $\mathrm{SF}_{6}$ filled spark gap, the capillary discharge section, and the diagnostic system have been completed. Experiments are presently being conducted to optimize the conditions first to obtain a short $\mathrm{X}$-ray pulse (1 ns) and then to look for the lasing properties like divergence and coherence, etc. We report here experimental details regarding generation of soft X-ray pulses of $\sim 5-10 \mathrm{~ns}$ duration.

The section 2 briefly describes the pinching mechanism and the generation of coherent $\mathrm{X}$-ray radiation via collision excitation mechanism in argon plasma. The details of this system are given in section 3. Electrical testing of the system has been completed and we report here the initial results in the X-ray emission from the capillary discharge. The results and discussions are presented in section 4 .

\section{Pinching mechanism and $\mathrm{X}$-ray lasing}

In the capillary discharge systems, a high voltage electrical discharge in the gas filled capillary produces plasma. Due to the high current flowing through the plasma, the $\mathrm{j} x \mathrm{~B}$ force in the plasma compresses it towards the axis of the capillary creating a hot, dense plasma channel of $100-200 \mu \mathrm{m}$ diameter. Under appropriate discharge conditions, lasing action can be obtained in the gas in the capillary.

Figure 1 shows the population inversion through collisional excitation mechanism in argon plasma. The lasing action is in the $\mathrm{Ar}^{8+}$ ions. As the temperature of the plasma increases, the abundance of $\mathrm{Ar}^{8+}$ ions (Ne-like) reaches its peak value between $\mathrm{T}_{e}=60$ and $80 \mathrm{eV}$ (BenKish et al 2001). The ground state configuration of $\mathrm{Ar}^{+8}$ is neon-like $\left(1 \mathrm{~s}^{2} 2 \mathrm{~s}^{2} 2 \mathrm{p}^{6}\right)$. The nearest excited states are: $1 s^{2} 2 s^{2} 2 p^{5} 3 s$ and $1 s^{2} 2 s^{2} 2 p^{5} 3 p$. Whereas the $3 s$ states have opposite parity, the $3 p$ states have same parity as the ground state. Hence optical transitions to and from the latter (3p) are forbidden, whereas those from 3 s level to and from ground state are optically allowed. Although collisional excitation from the ground state takes place to both the levels $(3 \mathrm{~s}, 3 \mathrm{p})$, the $3 \mathrm{~s}$ level gets quickly depopulated through fast radiative transition to the ground state, leading to population inversion in the $3 \mathrm{~s}$ and $3 \mathrm{p}$ states. Lasing can then take place between the $3 \mathrm{p}$ and 3 s states. This is the standard collisional inversion mechanism in neon-like ions. In the case of $\mathrm{Ar}^{+8}$, the lasing takes place at $46.9 \mathrm{~nm}$ wavelength $(26.5 \mathrm{eV})$.

It has been experimentally seen that due to various instabilities, the uniformity of this channel is poor, leading to irregular lasing action. In order to overcome this problem and to obtain reliable and uniform compression, a long pre-pulse is used to generate the pre-plasma and the main pulse 


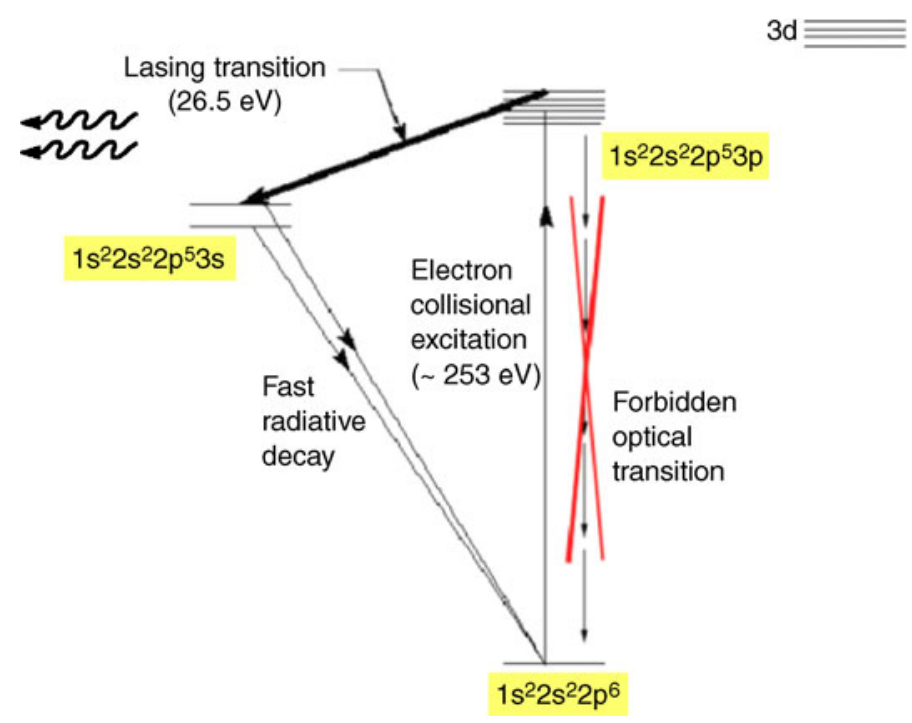

Figure 1. Collisional excitation based population inversion scheme in neon-like argon ions.

is used for compression of this preformed plasma (Ben-Kish et al 2001). The role of the prepulse in the laser action has been also studied by different groups in detail (Kolacek et al 2004). As one starts optimizing various parameters, a soft X-ray pulse starts appearing super imposed over broad pulse (pinching). The duration of this pulse reduces further and finally it leads to X-ray lasing.

\section{Experimental system}

The capillary discharge system at RRCAT (figures 2 and 3) consists of a $400 \mathrm{kV}, 750 \mathrm{~J}, 10$ stage Marx generator, a pulse forming line (PFL) in the form of a waterline capacitor $(\mathrm{C}=6 \mathrm{nF}, \mathrm{L}=$ $70 \mathrm{nH}$, and $\mathrm{Z}=3.3 \Omega$ ) to compress the current pulse in time, a spark gap filled with $\mathrm{SF}_{6}$ gas, and a ceramic (alumina) capillary $(2.8 \mathrm{~mm}$ dia), followed by an X-ray diagnostic system to carry out measurements. The density and temperature of the argon plasma varies rapidly when the fast varying current pulse $\left(\mathrm{dI} / \mathrm{dt}>10^{11} \mathrm{~A} / \mathrm{s}\right)$ is applied, and the magnetic field associated with this current compresses the plasma. In order to obtain a uniform compression, a $20 \mathrm{kV}$ electrical prepulse, whose current can be varied between 10 and $20 \mathrm{~A}$, is applied to precondition the plasma, 3-20 $\mu$ s before the main current pulse. In order to perform detailed parametric study of X-ray generation using this system, the control system had been fully automated. A micro-controller unit has been developed in-house, which can control the charging and firing the pre-pulse and main pulse generators. In addition, the voltages and the delay between the pre-pulse and the main pulse, can be varied from this controller to study the dependence of the X-ray emission on these parameters.

The pressure of the $\mathrm{SF}_{6}$ gas and the gap between the electrodes can be adjusted to vary the breakdown voltage, which in turn decides the capillary current. After the spark gap breaks down, the voltage appears across the capillary. The other end of the capillary is kept at ground potential. The adjacent chamber is used for adjusting and monitoring the argon gas pressure. The X-ray radiation passes through the aperture in the ground electrode and an orifice (dia $\sim 1 \mathrm{~mm}$ ) kept 


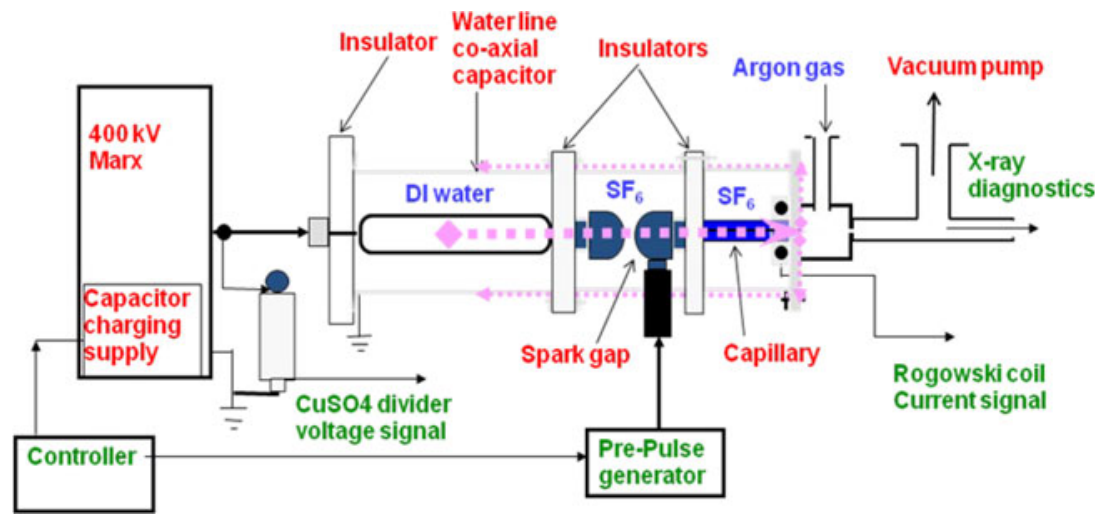

Figure 2. Schematic diagram of the capillary discharge set-up.

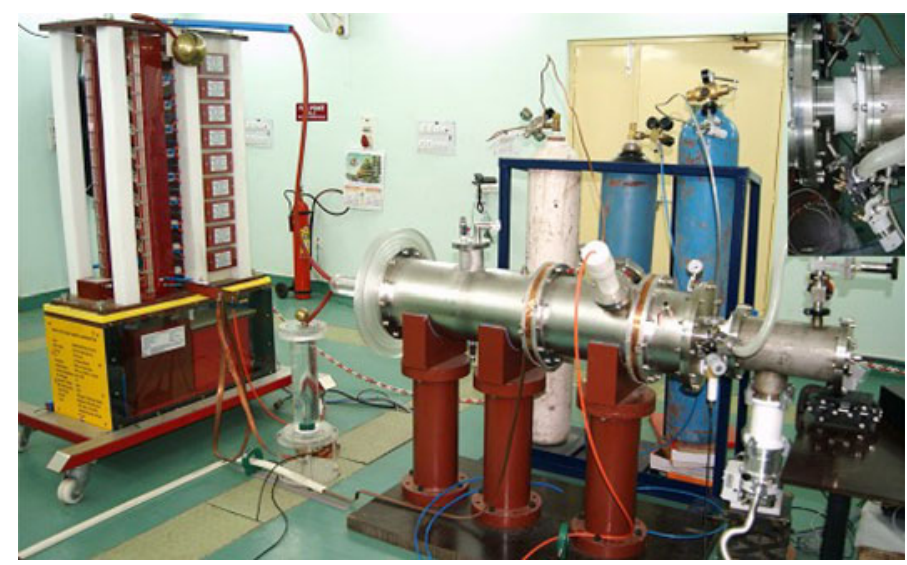

Figure 3. Experimental system (inset: close-up of the argon chamber).

at a small distance from the ground electrode (as the cold argon between the ground electrode and the orifice can absorb the XUV radiation). The orifice is used for maintaining differential pressure between the argon chamber and the diagnostics system. The latter needs to be kept at low pressure in order to reduce the absorption of soft X-rays. The diagnostics chamber is evacuated with the help of a turbo-molecular pumping system (TMP). The pressure of the argon gas in the capillary can be varied by adjusting the regulating inlet valve (Pfeiffer - EVN 116). The vacuum in the diagnostic system (buffer chamber onwards) varies between $10^{-6}$ and $10^{-4} \mathrm{mbar}$ when the gas pressure in the capillary is varied from $10^{-3}$ to 0.5 mbar. In addition, in order to ensure that the plasma channel (pinched) and the centre of the orifice are in line, the high voltage electrode, the capillary centre, the ground electrode and the orifice are aligned using a canon SLR digital camera, with an accuracy of 60 microns.

The voltage on the Marx generator was measured through a $\mathrm{CuSO}_{4}$ voltage divider. The current passing through ceramic capillary was measured using either an indigenously made Rogowskii coil, or a current transformer (CT) placed in the capillary chamber. Typical current and voltage profiles are shown in figure 4. For the initial experiments, the Marx generator was charged for $18 \mathrm{kV} /$ stage to get peak erected voltage $\sim 180 \mathrm{kV}$ corresponding to a current 


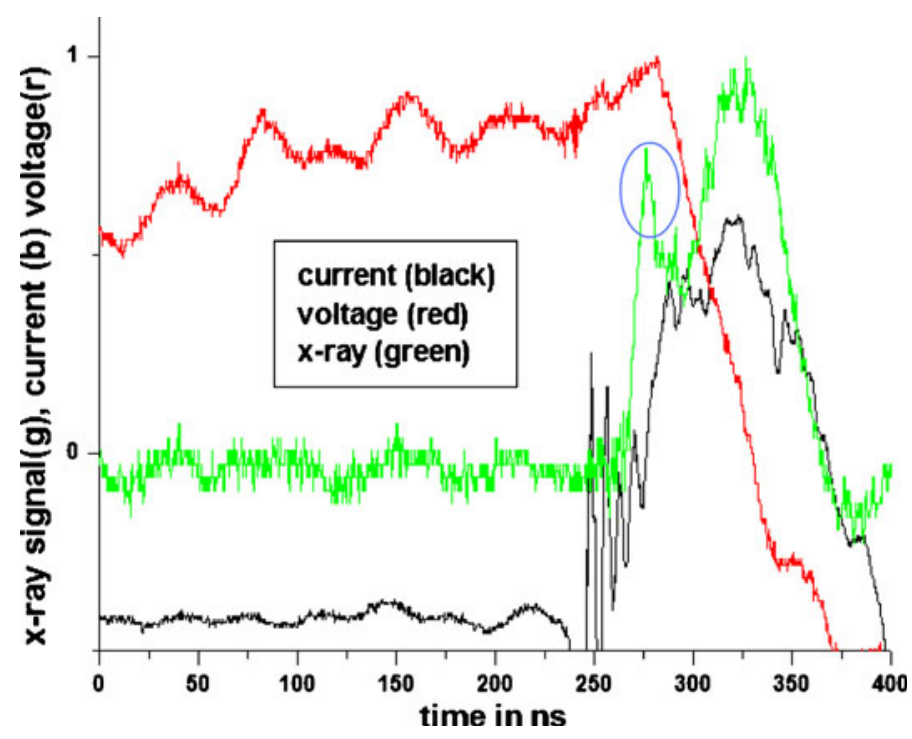

Figure 4. Current, voltage and X-ray signals (all normalized). Current pulse is vertically offset to show $\mathrm{X}$-ray signal clearly. The soft X-ray peak is circled.

of $\sim 20 \mathrm{kA}$. The noise appearing in the current pulse may be mainly due to the breakdown in the in spark gap/capillary section (fast component appearing at the beginning) and secondly due to oscillations between the stray capacitance/inductance in the capillary/spark gap (slow component). But it is expected that this can be minimized with better design of spark gap.

To detect the soft X-ray radiation and to ascertain lasing action (at $46.9 \mathrm{~nm}$, in the case of Ar gas), both, spectroscopic and time resolved studies are needed. As part of these X-ray laser studies, the X-ray radiation generated in the capillary channel was measured using two different diodes including IRD-AXUV diodes which are sensitive for the wavelength of our interest and Quantrad P-I-N-250 diodes. The IRD diodes were reverse biased at $150 \mathrm{~V}$ and the Quantrad diode was operated at $300 \mathrm{~V}$. A thin aluminium foil $(0.75 \mu \mathrm{m})$ was used to cover the IRD diodes in order to block the visible radiation from the plasma and transmit the X-rays.

\section{Results and discussion}

The emission from the capillary observed using the IRD diodes, consists of a short X-ray pulse, at a delay of $\sim 30 \mathrm{~ns}$ from the starting of current pulse $(>18 \mathrm{kA})$ as shown in the figure 4 . This pulse overrides a broad X-ray pulse which starts after $10 \mathrm{~ns}$. When the IRD diodes are replaced with Quantrad diodes, the XUV X-ray peak vanished, as the $0.75 \mu \mathrm{m}$ thick dead layer (glass) on these diodes absorbs the XUV X-rays. Only the broad pulse remained. Similarly, this short XUV signal was also absent when thick Al filters $(>2.4 \mu \mathrm{m})$ are used on the IRD diodes.

The pinching time can be calculated using a simple analytical 'snow-plow' model. In this model the pinch evolution is determined by simultaneous solution of dynamic external pumping circuit Kirchoff equations (treating the whole capillary system with a simple RLC circuit). Using this model the effect of experimental parameters like capillary current, voltage, gas pressure and capillary radius, etc., on the pinching time can be estimated. The collapse time (when the radius 
of the pinch goes to zero) in this case is expressed as (Vrba et al 2001, Krall \& Travelpiece 1973)

$$
\mathrm{t}_{\mathrm{c}}=1.5\left[\mathrm{ac} /(\mathrm{dI} / \mathrm{dt})_{\mathrm{o}}\right]^{1 / 2} \mathrm{M}_{\mathrm{o}}^{1 / 4}
$$

where a, I and $\mathrm{M}_{\mathrm{o}}$ represent the initial plasma radius, capillary current and the total mass of gas per unit length of plasma $\left(\pi \mathrm{a}^{2} \rho_{\mathrm{m}}\right)$ at the beginning of compression. For our experimental system at Laser Plasma Division, RRCAT, this model indicates a pinching time $\sim 35 \mathrm{~ns}$ (Ghomi et al 2007), which is in agreement with experiments. The measurements were repeated at different argon gas pressures in the range of $0.1-0.4$ mbar. The fact that the sharp X-ray pulse overriding the broad X-ray emission is absent when the same measurements are repeated with different Xray diodes (Quantrad vs IRD) or when thick filters are used on the IRD diodes indicates that the sharp pulse is due to soft X-rays and the broad peak is due to hard X-rays.

The duration of the present pulse varied between 5 and $10 \mathrm{~ns}$ (FWHM) in different shots. It is known that the amplitude of the pre-pulse and the main current, the delay between the two current pulses, and the gas pressure affect the pulse duration. When the pre-pulse current is above the optimum value, the pinching of the plasma is not efficient as the plasma is already heated. Similarly at lower main current values, the Bennet equilibrium (which describes the equilibrium between the magnetic force which acts inwards and the outward plasma kinetic force) occurs at a larger plasma radius, which leads to longer and less intense soft X-ray pulse.

It must be mentioned here that the main hurdle in these measurements was the presence of large electro-magnetic interference/coupling (EMI/EMC) due to the presence of the fast rising high voltages and large currents. This resulted in appearance of voltages of few kilovolts on metallic parts in the diagnostic system. This coupling can be both capacitive and inductive couplings in nature since the voltages are very high (hundreds of $\mathrm{kV}$ ) and the currents are also fast varying in time $\left(\mathrm{dI} / \mathrm{dt}>10^{11} \mathrm{~A} / \mathrm{s}\right)$. In order to minimize these effects, the cross-sectional areas of the diagnostics systems components are kept to minimum possible and the distance was also increased. An insulating spacer made of teflon was inserted between the capillary chamber and the buffer chamber. Similarly, another teflon insulating spacer was inserted between the buffer chamber and the TMP system to protect the latter. In addition, isolation transformers had been used in the electrical lines (for Marx bank, pre-pulse and TMP) and all the ground loops had been eliminated by using separate ground pits for the Marx bank and diagnostics side. It was seen during the experiments that maintaining proper vacuum levels in the diagnostics section was also very crucial. Moreover, all the connecting cables of the sensitive equipment like vacuum gauges are disconnected to protect them before firing the Marx bank. In addition, shielded cables have been used to carry the X-ray signals (few volts signal) from the X-ray diode up to the Faraday cage, where the oscilloscopes and controlling system were kept to protect from radiative noise generated from the Marx bank and spark gaps. Only after overcoming these EMI problems, the $\mathrm{X}$-ray emission measurements could be made.

\section{Conclusions}

The present measurements have shown production of a short XUV X-ray pulse in the argon filled capillary discharge, which appears to be due to pinching of the argon plasma by the fast varying current pulse. However, as discussed in the results and discussion section, the present measurements only indicate the initial phase of the study and after optimization of the current pulses (both amplitude and delay between them) and the filling pressure of the argon gas, we expect the pulse duration which is presently $\sim 5-10 \mathrm{~ns}$ to become further narrower (to reach $\sim 1 \mathrm{~ns}$ ). Measurements of other parameters like monochromacity, divergence, etc. of the X-ray beam will 
have to be carried out after this optimization to look for X-ray lasing. Future measurements will include the spectroscopic study, and X-ray emission measurements at longer distances. Efforts will also be made to increase the current flowing through the capillary channel beyond $20 \mathrm{kA}$, and study its effect on the X-ray generation.

The authors would like to thank Mr. R P Kushwaha for all the valuable support and cooperation extended for this study.

\section{References}

Ben-Kish A, Shuker M, Nemirovsky R A, Fisher A, Ron A and Schwob J L 2001 Plasma dynamics in capillary discharge soft X-ray lasers. Phys. Rev. Lett. 87: 015002-1-4

Filevich J, Kanizay K, Marconi M C, Chilla J L A, and Rocca J J 2000 Dense plasma diagnostics with an amplitude-division soft-X-ray laser interferometer based on diffraction gratings. Opt. Let. 25: 356-358

Filevich J, Rocca J J, Marconi M C, Smith RF, Dunn J, Keenan R, Hunter J R, Moon S J, Nilsen J, Ng A, and Shlyaptsev V N 2004 Picosecond-resolution soft-X-ray laser plasma interferometry. Appl. Optics 43: 3938-3946

Ghomi H, Rostami S and Latifi H 2007 Study of Z-pinch evolution by snow-plow model Proceedings of $28^{\text {th }}$ ICPIG, July 15-20, 2007, Prague, Czech Republic: 558-560

Gupta P D, Naik P A 2008 Ultra-intense Laser Plasma Interaction Studies at RRCAT, Indore. Rev. Laser Eng. 36: 1119-1122

Hara T, Ando K, Kusakabe N, Yashiro H and Aoyagi Y 1989 Soft X-ray lasing in an Al Plasma produced by a 6 J laser. Jap. J. App. Phys. 28: L1010-L1012

Heinbuch S, Grisham M, Martz D and Rocca J J 2005 Demonstration of a desk-top size high repetition rate soft X-ray laser. Optics Express 13: 4050-4055

http://en.wikipedia.org/wiki/Extreme_ultraviolet_lithography,

http://www.physorg.com/news123256413.html

Kolacek K, Schmidt J, Pruckner V, Straus J, Bohacek V, Frolov O and Vrba P 2004 Role of pre-pulse in gas filled capillary soft X-ray source. Czechoslovak. J. of Phys. 54, Supplement C: C334-C343

Krall N A and Trivelpiece A W 1973 Principles of plasma physics (McGraw-Hill) 123-127

Pellegrini C, Stohr J 2003 X-ray free-electron lasers-principles, properties and applications. Nucl. Instrum. \& Meth. in Phys. Res. A 500: 33-40

Rocca J J, Filevich J, Hammarsten E C, Jankowska E, Benware B, Marconi M C, Luther B, Vinogradov A, Artiukov I, Moon S, Shlyaptsev V N 2003 Extremely compact soft X-ray lasers based on capillary discharges. Nucl. Instrum. \& Meth. in Phys. Res. A 507: 515-522

Salieres P, Lewenstein M 2001 Generation of ultrashort coherent XUV pulses by harmonic conversion of intense laser pulses in gases: towards attosecond pulses. Measure Sci. and Technol. 12: 1818-1827

Smith R, Tallents G J, Zhang J, Eker G, McCabe S, Pert G J, Wolfrum E 1999 Saturation behavior of two $\mathrm{x}$-ray lasing transitions in Ni-like Dy. Phys. Rev. A 59: R47-R50

Suckewer S, Jaegl'e P 2009 X-ray laser: past, present, and future. Las. Phys. Lett. 6: 411-436

Tallents G J 2003 The physics of soft X-ray lasers pumped by electron collisions in laser plasmas. J. Phys. D: Appl. Phys. 36: R259-R276

Tan C A, Kwek K H 2007 Influence of current prepulse on capillary-discharge extreme-ultraviolet laser. Phys. Rev. A 75: 043808-1-5

Vrba P, Vrbová M, Kálal M, Janacąárek A and Fojtík A 2001 Role of initial vapor density in Z-pinch polyacetal capillary discharge. NUKLEONIKA 46: S2-S27 